\title{
COMPOSIÇÃO FLORÍSTICA DA FLORESTA OMBRÓFILA MISTA MONTANA DO PARQUE MUNICIPAL DO BARIGÜI, CURITIBA, PR ${ }^{1}$
}

\author{
Carina Kozera*, Vinícius Antonio de Oliveira Dittrich**, Sandro Menezes Silva*** \\ * Bióloga, M.Sc., Doutoranda em Eng. Florestal, UFPR - kozera23@yahoo.com \\ **Biólogo, Dr., UNICSUL - vinarc@gmail.com \\ ***Biólogo, Dr. - epifita@terra.com.br
}

Recebido para publicação: 10/01/2006 - Aceito para publicação: 27/04/2006

\begin{abstract}
Resumo
Realizou-se o levantamento florístico das espécies vasculares (exceto epífitas) de um fragmento de Floresta Ombrófila Mista Montana localizado no Parque Municipal do Barigüi, município de Curitiba, PR. Foram registradas 390 espécies, 226 gêneros e 99 famílias, entre pteridófitas, gimnospermas e angiospermas. Dentre as pteridófitas, destacaram-se como as mais ricas Dryopteridaceae (oito) e Blechnaceae (seis); e dentre as angiospermas, Asteraceae (30), Solanaceae (25), Myrtaceae (25) e Poaceae (17). As gimnospermas foram representadas por somente duas famílias, Araucariaceae e Podocarpaceae, cada uma delas com uma espécie. Foram listadas 141 espécies arbóreas, 129 herbáceas, 67 arbustivas, 50 trepadeiras e três hemiepífitas. A floresta, apesar de situada dentro de uma zona urbana, sujeita à intensa interferência antrópica, apresentou elevada riqueza específica.

Palavras-chave: Floresta com Araucária; Floresta Ombrófila Mista; Araucaria angustifolia; levantamento florístico; parque municipal.
\end{abstract}

\begin{abstract}
Floristic composition of the Mixed Ombrophilous Montane Forest at Barigüi County Park, Parana, Brazil. The checklist (except epiphytes) of a subtropical ombrophilous montane forest at Barigüi Park, Curitiba County, listed 390 species, 226 genera and 99 families (pteridophytes, gymnosperms and angiosperms). The best-represented pteridophytes families were Dryopteridaceae (eight species) and Blechnaceae (six species) the angiosperms were Asteraceae (30 species), Solanaceae ( 25 species), Myrtaceae (25 species) and Poaceae (17 species). The gymnosperms were represented by two families only (Araucariaceae and Podocarpaceae) each one with one specie. We listed 141 trees, 129 herbs, 67 bushes, 50 vines and tree hemiepiphytes. Even thought being inside the city and showing significant anthropic influence the forest had a high floristic diversity.

Keywords: Araucaria Forest; subtropical ombrophilous forest; Araucaria angustifolia; floristic survey; county park.
\end{abstract}

\section{INTRODUÇÃO}

Floresta com Araucária (HUECK, 1953), Pinheiral (RIZZINI et al., 1988) e Floresta Ombrófila Mista (VELOSO et al., 1991) são algumas denominações utilizadas na literatura para se referir às formações florestais caracterizadas pela presença de Araucaria angustifolia (Bertol.) Kuntze Araucariaceae, espécie arbórea também conhecida como pinheiro-do-paraná ou pinheiro-brasileiro.

Com copas corimbiformes e folhagem verde-escuro, essa espécie encontra-se na floresta geralmente representada por indivíduos emergentes, os quais imprimem um aspecto fitofisionômico próprio e muito característico à Floresta Ombrófila Mista (FOM). Abaixo dos indivíduos emergentes podem ainda ser observados outros três estratos, o arbóreo superior, o arbóreo inferior e o arbustivoherbáceo (KLEIN, 1979).

\footnotetext{
${ }^{1}$ Parte do projeto de pesquisa "Estudo florístico e fitossociológico da vegetação do Parque Barigüi, Curitiba, PR" (BANPESQ 95003945).
} 
A FOM compreende as formações florestais típicas dos planaltos da região Sul do Brasil, com disjunções na região Sudeste e em países vizinhos (Argentina e Paraguai). Encontra-se predominantemente entre 800 e $1200 \mathrm{~m}$ s.n.m., podendo eventualmente ocorrer acima desses limites (RODERJAN et al., 2002). As áreas ocupadas pela floresta apresentam valores de precipitação média situados entre 1500 e $1750 \mathrm{~mm}$ anuais e temperatura variável, sendo que no verão as médias estão entre $20^{\circ}$ e $21^{\circ} \mathrm{C}$ e no inverno entre $10^{\circ}$ e $11^{\circ} \mathrm{C}$ (KLEIN, 1960). De acordo com IBGE (1992), a FOM pode ser subdividida e classificada em formação Aluvial, Submontana, Montana e Altomontana, em função da latitude e altitude de ocorrência da vegetação.

$\mathrm{Na}$ última década do século XX, as áreas ocupadas pela FOM no sul do Brasil foram bastante reduzidas. A exploração madeireira de Araucaria angustifolia e de espécies consorciadas a ela, como por exemplo a imbuia (Ocotea porosa (Nees) L. Barr.), e a expansão de áreas agrícolas representam alguns dos fatores responsáveis pela expressiva redução da área ocupada por esse tipo vegetacional (BACKES, 1983).

Considerando-se a atual realidade da devastação das florestas brasileiras, trabalhos de levantamento qualitativo e/ou quantitativo destacam-se em importância no processo de conhecimento da flora e da estrutura da vegetação. Eles contribuem para a compreensão da dinâmica das comunidades vegetacionais (RODRIGUES, 1988) e são fundamentais em trabalhos de recuperação de áreas degradadas.

No Brasil, especialmente na região Sul e em áreas de FOM, trabalhos dessa natureza foram realizados por Dombrowski e Kuniyoshi (1967), Klein (1963, 1979), Hatschbach e Moreira Filho (1972), Longhi (1980), Oliveira e Rotta (1980), Martau et al. (1981), Cestaro et al. (1986), Jarenkow e Baptista (1987), Cervi et al. (1987a, 1987b, 1988, 1989), Galvão et al. (1989), Roseira (1990), Silva e Marconi (1990), Machado et al. (1992), Negrelle e Silva (1992), Britez et al. (1995), Senna e Waechter (1997), Silva et al. (1997, 1998) e Dittrich et al. (1999). Contribuíram, entre outros aspectos, para o melhor entendimento e conhecimento das espécies presentes em florestas sulinas do país.

Com esse mesmo intuito, realizou-se o levantamento florístico de um fragmento de FOM localizado em um parque municipal de Curitiba. Os objetivos do trabalho foram listar as espécies vasculares (exceto epífitas) e classificá-las quanto à forma de vida. O trabalho justifica-se pelo desconhecimento da flora do Parque e pela importância do fragmento em termos de representatividade das florestas que ocupavam, em décadas passadas, grande parte do município. O conhecimento da flora contribuirá para o desenvolvimento de atividades de educação ambiental direcionadas para os moradores, visitantes e estudantes de diferentes níveis de escolaridade, bem como poderá subsidiar projetos com finalidade de conservação da área.

\section{MATERIAL E MÉTODOS}

O Parque Municipal do Barigüi é uma das maiores áreas verdes da região urbana do município de Curitiba. É um dos parques municipais mais visitados e freqüentados pelos habitantes da cidade, encontrando-se, por esse motivo, sob pressão de diferentes atividades antrópicas, tais como a intensa circulação de pessoas e a dispersão de espécies exóticas e/ou ruderais. Está situado a $900 \mathrm{~m}$ s.n.m. e apresenta cerca de 140 ha (Paraná, 1994). Desse total, aproximadamente 70 ha estão ocupados por jardins, lagos e áreas construídas para a recepção de visitantes (lanchonetes, estacionamentos, quadras esportivas, calçadões e ciclovias), e 70 ha ocupados por quatro diferentes tipologias de vegetação: Floresta Ombrófila Mista Montana, Floresta Ombrófila Mista Aluvial, Formação Pioneira com Influência Fluvial e Sistemas de Vegetação Secundária (ABE et al., 2000).

O levantamento florístico foi realizado no fragmento de Floresta Ombrófila Mista Montana. Essa tipologia ocupa cerca de 17,5 ha do Parque (ABE et al., 2000) e foi escolhida para o trabalho por ocupar uma área contínua e em melhor estado de conservação, quando comparada com as demais.

A área, segundo a classificação climática de Köppen, está inserida em uma região com o tipo $\mathrm{Cfb}$, subtropical úmido, mesotérmico, com verões frescos e geadas severas, sem estação seca (IAPAR, 1978). A temperatura média anual é de $16,7^{\circ} \mathrm{C}$, sendo a média mensal no mês mais quente (fevereiro) igual a $20,5^{\circ} \mathrm{C}$, e no mês mais frio (julho) igual a $13,0^{\circ} \mathrm{C}$. A precipitação média mensal varia entre $75 \mathrm{e}$ $169 \mathrm{~mm}$ (ROSEIRA, 1990). Quanto aos solos, destacam-se como classes predominantes, nas áreas não hidromórficas, os Cambissolos e Argissolos, e, nas áreas mais hidromórficas, os Gleissolos. 
Para o levantamento, foram realizadas caminhadas semanais no interior e entorno do fragmento, no período entre 1995 e 1997. Os procedimentos de coleta e herborização seguiram as técnicas citadas em IBGE (1992). Para a determinação, utilizou-se bibliografia específica para as famílias, gêneros e espécies, comparações com exsicatas dos Herbários UPCB (Departamento de Botânica, Universidade Federal do Paraná) e MBM (Museu Botânico Municipal de Curitiba) e consultas a especialistas que trabalham com taxonomia vegetal. Todo o material coletado, devidamente herborizado e determinado, foi registrado no Herbário UPCB. Foram incluídas espécies de pteridófita, gimnosperma e angiosperma.

Quanto às formas de vida, as espécies foram classificadas em: arbórea (planta terrícola, lenhosa, com tronco principal e, geralmente, com altura superior a $5 \mathrm{~m}$ ), arbustiva (planta terrícola, lenhosa, sem tronco principal, geralmente ramificada desde a base e com altura inferior a $5 \mathrm{~m}$ ), herbácea (planta terrícola e não lenhosa), trepadeira (planta terrícola, herbácea ou lenhosa, com estruturas para fixação, volúveis ou apoiantes, desenvolvendo-se geralmente sobre outras espécies vegetais ou superfícies de apoio) e hemiparasita (planta que cresce sobre outras espécies vegetais mantendo contato nutricional com elas através de haustórios, estruturas especializadas para absorção de seiva) (FONT QUER, 1963). As espécies epifíticas (plantas que crescem sobre outras espécies vegetais, sem manter contato com estas para a absorção de nutrientes) foram observadas e coletadas no Parque durante o mesmo período, porém apresentadas à parte por Dittrich et al. (1999).

Para o nome dos autores das espécies adotaram-se as abreviaturas propostas por Brummitt e Powell (1992), e para a conferência da escrita dos nomes científicos foram consultados os bancos de dados eletrônicos do Jardim Botânico de Missouri (Missouri Botanical Garden, 2006) e do IPNI (The International Plant Names Index, 2006).

A listagem das pteridófitas foi apresentada com base no sistema de classificação proposto por Tryon e Tryon (1982), e a das angiospermas, no de Cronquist (1988). Optou-se por esses sistemas pelo fato de serem utilizados no herbário onde os materiais foram depositados.

\section{RESULTADOS}

Foram registradas 342 espécies de angiospermas, 46 de pteridófitas e duas gimnospermas, totalizando 390 espécies. Dessas, 12 foram determinadas até o nível de gênero e quatro até o nível de família.

As pteridófitas foram representadas por 14 famílias (Tabela 1), com destaque para Dryopteridaceae (8), Blechnaceae (6), Aspleniaceae (5) e Thelypteridaceae (5); as angiospermas, por 83, com destaque para Asteraceae (30), Solanaceae (25), Myrtaceae (25), Poaceae (17) e Rubiaceae (13). As gimnospermas estiveram presentes na floresta representadas por somente duas espécies de diferentes famílias: Araucaria angustifolia - Araucariaceae, e Podocarpus lambertii Klotzsch ex Endl. Podocarpaceae (Tabela 2).

Do total de espécies registradas, aproximadamente 36\% (141) apresentaram forma de vida arbórea, 33\% (129) herbácea, 17\% (67) arbustiva, 13\% (50) trepadeira e menos de um por cento (3) hemiparasita.

As arbóreas foram representadas principalmente por espécies de Myrtaceae (23), Lauraceae (11), Aquifoliaceae (7) e Flacourtiaceae (7). Os arbustos, por Solanaceae (16) e Asteraceae (14); e as herbáceas, por Poaceae (17), Cyperaceae (8), Dryopteridaceae (8), Asteraceae (6) e Blechnaceae (6). Dentre as herbáceas, importante destacar a contribuição das espécies de pteridófita na diversidade da floresta. Do total de espécies herbáceas registradas, $32 \%$ (41) pertencem a essa divisão.

Com relação às trepadeiras, Passifloraceae (6) e Sapindaceae (5) são as famílias mais ricas em espécies com essa forma de vida. A primeira foi representada principalmente por trepadeiras herbáceas, e a segunda, por trepadeiras lenhosas. As hemiparasitas foram registradas somente em Loranthaceae.

No interior e entorno da floresta, principalmente próximo aos caminhos de circulação de pessoas, foram identificadas algumas espécies exóticas, com base nos trabalhos de Lorenzi (1982), Graf (1992) e Lorenzi e Souza (1995). Dentre essas, citam-se: Centella asiatica (L.) Urb., Tetrapanax papyriferum K. Koch, Impatiens walleriana Hook.f., Hovenia dulcis Thunb., Eryobotrya japonica Lindl., Lantana camara L., Setaria poiretiana (Schult.) Kunth, Physalis pubescens L., Crocosmia crocosmiflora (Nicholson) N. E. Br. e Lonicera japonica Thunb. 
Tabela 1. Pteridófitas da Floresta Ombrófila Mista Montana do Parque Municipal do Barigüi (Curitiba, PR), com informações sobre a forma de vida observada em campo (ab-arbustiva, he-herbácea), mês de coleta e o número de registro no Herbário do Departamento de Botânica da Universidade Federal do Paraná (UPCB). s/col. = sem coleta de material fértil.

Table 1. Pteridophytes of the Montane Ombrophilous Mixed Forest at Barigüi Park (Curitiba, Paraná, Brazil), showing informations about the field observed life form (ab - shrub, he - herb), collection month, and register number at the Herbarium of the Universidade Federal do Paraná Botany Department (UPCB). s/col.= with no fertile material collected.

\begin{tabular}{|c|c|c|c|c|}
\hline Famílias & Espécies & $\begin{array}{l}\text { Forma } \\
\text { de vida }\end{array}$ & Mês & UPCB \\
\hline \multirow{5}{*}{ Aspleniaceae } & Asplenium claussenii Hieron. & he & fev & 39638 \\
\hline & Asplenium gastonis Fée & he & out & 26030 \\
\hline & Asplenium harpeodes Kunze & he & jan & 27399 \\
\hline & Asplenium inequilaterale Willd. & he & mar & 28058 \\
\hline & Asplenium pseudonitidum Raddi & he & mai & 28057 \\
\hline \multirow[t]{6}{*}{ Blechnaceae } & Blechnum austrobrasilianum de La Sota & he & mai & 28389 \\
\hline & Blechnum brasiliense Desv. & he & jun & 27388 \\
\hline & $\begin{array}{l}\text { Blechnum binervatum ssp. acutum (Desv.) R. M. Tryon e } \\
\text { Stolze }\end{array}$ & he & out & 28388 \\
\hline & Blechnum confluens Cham. e Schltdl. & he & mai & 29674 \\
\hline & Blechnum cordatum (Desv.) Hieron. & he & jul & 40812 \\
\hline & Blechnum schomburgkii (Klotzsch) C. Chr. & he & jul & 40810 \\
\hline \multirow[t]{4}{*}{ Cyatheaceae } & Alsophila setosa Kaulf. & $\mathrm{ab}$ & jan & 26031 \\
\hline & Cyathea delgadii Sternb. & $\mathrm{ab}$ & fev & 29668 \\
\hline & Cyathea corcovadensis (Raddi) Domin & $a b$ & mai & 26061 \\
\hline & Cyathea phalerata Mart. & $a b$ & out & 29678 \\
\hline Davalliaceae & Nephrolepis occidentalis Kunze & he & dez & 30586 \\
\hline \multirow[t]{3}{*}{ Dennstaedtiaceae } & Dennstaedtia dissecta (Sw.) Moore & he & mar & 29285 \\
\hline & Hypolepis repens (L.) C. Presl & he & dez & 39370 \\
\hline & Lindsaea botrychioides A. St.-Hil. & he & fev & 26062 \\
\hline Dicksoniaceae & Dicksonia sellowiana Hook. & $\mathrm{ab}$ & set & 28375 \\
\hline \multirow{8}{*}{ Dryopteridaceae } & Ctenitis anniesii (Rosenst.) Copel. & he & mai & 28573 \\
\hline & Ctenitis falciculata (Raddi) Ching & he & mar & 29676 \\
\hline & Ctenitis submarginalis (Langsd. e Fisch.) Ching & he & set & 28572 \\
\hline & Didymochlaena truncatula (Sw.) J. Smith & he & - & $\mathrm{s} / \mathrm{col}$ \\
\hline & Lastreopis amplissima (C. Presl) Tindale & he & jun & 29831 \\
\hline & Megalastrum connexum (Kaulf.) A. R. Sm. e R. C. Moran & he & nov & 30584 \\
\hline & Polystichum montevidense (Spreng.) Rosenst. & he & nov & 39636 \\
\hline & Polystichum platylepis Fee & he & jun & 27398 \\
\hline \multirow[t]{2}{*}{ Gleicheniaceae } & Dicranopteris flexuosa (Schrad.) Underw. & he & mar & 39369 \\
\hline & Sticherus penniger (Mart.) Copel. & he & nov & 28574 \\
\hline Marattiaceae & Marattia laevis Sm. & he & dez & 29286 \\
\hline \multirow[t]{3}{*}{ Polypodiaceae } & Pecluma pectinatiformis (Lindm.) M. G. Price & he & out & 28423 \\
\hline & Polypodium catharinae Langsd. e Fisch. & he & mai & 27396 \\
\hline & Polypodium latipes Langsd. e Fisch. & he & jan & 26063 \\
\hline \multirow[t]{2}{*}{ Pteridaceae } & Adiantum raddianum C. Presl & he & mai & 27390 \\
\hline & Cheilanthes regularis Mett. & he & mar & 29439 \\
\hline \multirow[t]{2}{*}{ Pteridaceae } & Pteris deflexa Link & he & jun & 26037 \\
\hline & Pteris lechleri Mett. & he & jan & 25979 \\
\hline \multirow[t]{2}{*}{ Schizaeaceae } & Anemia flexuosa (Savigny) Sw. & he & mai & 27395 \\
\hline & Anemia phyllitidis (L.) Sw. & he & out & 25636 \\
\hline & Selaginella sulcata (Desv. ex Poir.) Spring ex Mart. & he & mai & 27394 \\
\hline \multirow{5}{*}{ Thelypteridaceae } & Thelypteris araucariensis Ponce & he & nov & 29685 \\
\hline & Thelypteris dentata (Forssk.) E. P. St. John & he & abr & 29679 \\
\hline & Thelypteris interrupta (Willd.) Iwatsuki & he & mar & 29683 \\
\hline & Thelypteris riograndensis (Lindm.) C. F. Reed & he & mai & 27391 \\
\hline & Thelypteris sp. & he & fev & 39909 \\
\hline
\end{tabular}


Tabela 2. Gimnospermas e angiospermas da Floresta Ombrófila Mista Montana do Parque Municipal do Barigüi (Curitiba, PR), com informações sobre a forma de vida observada em campo (avarbórea, ab-arbustiva, he-herbácea, tr-trepadeira, hp-hemiparasita), mês de coleta e o número de registro no Herbário do Departamento de Botânica da Universidade Federal do Paraná (UPCB). s/col. = sem coleta de material fértil.

Table 2. Gymnosperms and flowering plants of the Montane Ombrophilous Mixed Forest at Barigüi Park (Curitiba, Paraná, Brazil), showing informations about field observed life form (av-tree, ab-shrub, he-herb, tr-vine, hp-hemiparasite), collection month, and register number at the Herbarium of the Universidade Federal do Paraná Botany Department (UPCB). s/col.= with no fertile material collected.

\begin{tabular}{|c|c|c|c|c|}
\hline Famílias & Espécies & $\begin{array}{l}\text { Forma } \\
\text { de vida }\end{array}$ & Mês & UPCB \\
\hline & GIMNOSPERMA & & & \\
\hline Araucariaceae & Araucaria angustifolia (Bertol.) Kuntze & av & jan & 25981 \\
\hline \multirow[t]{2}{*}{ Podocarpaceae } & Podocarpus lambertii Klotzsch ex Endl. & av & jan & 25982 \\
\hline & ANGIOSPERMA & & & \\
\hline \multirow{3}{*}{ Acanthaceae } & Justicia carnea (Lindl.) G. Nicholson & $\mathrm{ab}$ & out & 28383 \\
\hline & Justicia floribunda (C. Koch) Wassh. & $a b$ & set & 29288 \\
\hline & Ruellia brevifolia (Pohl) C. Ezcurra & he & abr & 28030 \\
\hline \multirow[t]{2}{*}{ Amaranthaceae } & Chamissoa acuminata Mart. & he & nov & 29291 \\
\hline & Iresine diffusa Humb. e Bonpl. ex Willd. & he & mar & 30307 \\
\hline \multirow{3}{*}{ Anacardiaceae } & Lithraea brasiliensis Marchand & av & out & 28384 \\
\hline & Schinus polygamus (Cav.) Cabrera & $\mathrm{ab}$ & ago & 27099 \\
\hline & Schinus terebinthifolius Raddi & av & jan & 25987 \\
\hline \multirow[t]{2}{*}{ Annonaceae } & Guatteria australis A. St.-Hil. & av & - & $\mathrm{s} / \mathrm{col}$ \\
\hline & Rollinia emarginata Schltdl. & av & out & 28581 \\
\hline Apiaceae & Centella asiática (L.) Urb. & he & out & 29292 \\
\hline \multirow{2}{*}{ Apocynaceae } & Forsteronia velloziana (A.DC.) Woodson & $\operatorname{tr}$ & out & 27097 \\
\hline & Peltastes peltatus (Vell.) Woodson & $\operatorname{tr}$ & mai & 30656 \\
\hline \multirow[t]{7}{*}{ Aquifoliaceae } & Ilex amara Loes. & av & - & $\mathrm{s} / \mathrm{col}$ \\
\hline & Ilex brevicuspis Reissek & av & - & $\mathrm{s} / \mathrm{col}$ \\
\hline & Ilex dumosa Reissek & av & mai & 27098 \\
\hline & Ilex integerrima (Vell.) Reissek & av & mar & 30095 \\
\hline & Ilex paraguariensis A. St.-Hil. & av & out & 28582 \\
\hline & Ilex theezans Mart. & av & - & $\mathrm{s} / \mathrm{col}$ \\
\hline & Ilex sp. & av & - & $\mathrm{s} / \mathrm{col}$ \\
\hline \multirow[t]{2}{*}{ Araceae } & Asterostigma lividum (Lodd.) Engl. & he & out & 28580 \\
\hline & Spathicarpa hastifolia Hook. & he & out & 28583 \\
\hline Araliaceae & Tetrapanax papyriferum $\mathrm{K}$. Koch & av & - & s/col. \\
\hline Arecaceae & Syagrus romanzoffiana (Cham.) Glassman & av & - & $\mathrm{s} / \mathrm{col}$ \\
\hline \multirow[t]{4}{*}{ Asclepiadaceae } & Orthosia urceolata P. Fourn. & $\operatorname{tr}$ & out & 28310 \\
\hline & Oxypetalum wightianum Hook. & $\operatorname{tr}$ & jan & 27101 \\
\hline & Tassadia subulata (Vell.) Fontella e E. A. Schwarz & $\operatorname{tr}$ & mai & 27440 \\
\hline & Araujia sericofera Brot. & $\operatorname{tr}$ & fev & 40808 \\
\hline \multirow[t]{11}{*}{ Asteraceae } & Adenostemma verbesina (L.) Sch. Bip. & he & jan & 27442 \\
\hline & Baccharis anomala DC. & $\mathrm{ab}$ & jan & 30096 \\
\hline & Baccharis brachylaenoides DC. var. brachylaenoides & $\mathrm{av}$ & set & 39649 \\
\hline & $\begin{array}{l}\text { Baccharis brachylaenoides DC. var. polycephala (Schultz- } \\
\text { Bip.) G. M. Barroso }\end{array}$ & av & set & 39649 \\
\hline & Baccharis dracunculifolia DC. & $\mathrm{ab}$ & set & 39661 \\
\hline & Baccharis erioclada DC. & $\mathrm{ab}$ & nov & 29299 \\
\hline & Baccharis helichrysoides A.DC. & $\mathrm{ab}$ & mai & 31454 \\
\hline & Baccharis punctulata DC. & $\mathrm{ab}$ & dez & 30097 \\
\hline & $\begin{array}{l}\text { Baccharis semiserrata DC. var. elaeagnoides (Steud. ex } \\
\text { Baker) G. M. Barroso }\end{array}$ & $\mathrm{ab}$ & set & 26066 \\
\hline & Baccharis vincaefolia Baker & $\mathrm{ab}$ & jul & 25640 \\
\hline & Blainvillea biaristata DC. & he & jan & 27443 \\
\hline
\end{tabular}




\begin{tabular}{|c|c|c|c|c|}
\hline Famílias & Espécies & $\begin{array}{l}\text { Forma } \\
\text { de vida }\end{array}$ & Mês & UPCB \\
\hline & Chaptalia nutans (L.) Pol. & he & mai & 27444 \\
\hline & Conyza notobellidiastrum Griseb. & he & mai & 27445 \\
\hline & Conyza rivularis Gardner & he & ago & 40806 \\
\hline & Dasyphyllum sp. & $\mathrm{ab}$ & - & s/col. \\
\hline & Eupatorium inulaefolium Humb., Bonpl. e Kunth & he & jan & 28034 \\
\hline & Eupatorium serratum Spreng. & $\mathrm{ab}$ & nov & 30099 \\
\hline & $\begin{array}{l}\text { Eupatorium vauthierianum DC. var. ramosissimum (Gardner) } \\
\text { Baker }\end{array}$ & $\mathrm{ab}$ & out & 28035 \\
\hline & Gochnatia polymorpha (Less.) Cabrera & av & mar & 30100 \\
\hline & Mikania burchellii Baker & $\operatorname{tr}$ & set & 27446 \\
\hline & Mikania chlorolepis Baker & $\operatorname{tr}$ & mar & 30102 \\
\hline & Mikania ligustrifolia DC. & $\operatorname{tr}$ & set & 25641 \\
\hline & Mikania micrantha Humb. Bonpl. e Kunth & $\operatorname{tr}$ & mar & 27107 \\
\hline & Piptocarpha angustifolia Dusén & av & jan & 29662 \\
\hline & Piptocarpha axillaris (Less.) Baker & av & mai & 26071 \\
\hline & Polymnia connata (Spreng.) Blake & $\mathrm{ab}$ & jan & 28068 \\
\hline & Senecio pluricephalus Cabrera & $\mathrm{ab}$ & mar & 28036 \\
\hline & Vernonia discolor (Spreng.) Less. & av & out & 28315 \\
\hline & Vernonia puberula Less. & $\mathrm{ab}$ & mai & 27447 \\
\hline & Vernonia quinqueflora Less. & $\mathrm{ab}$ & jul & 28037 \\
\hline Balsaminaceae & Impatiens walleriana Hook.f & he & ago & 26039 \\
\hline \multirow[t]{2}{*}{ Begoniaceae } & Begonia fischeri Schrank & he & fev & 27102 \\
\hline & Begonia fruticosa (Klotzsch) A. DC. & $\operatorname{tr}$ & $\mathrm{abr}$ & 30575 \\
\hline Berberidaceae & Berberis laurina Billb. & $a b$ & set & 28351 \\
\hline \multirow[t]{6}{*}{ Bignoniaceae } & Arrabidaea selloi (Spreng.) Sandwith & $\operatorname{tr}$ & jan & 28032 \\
\hline & Jacaranda puberula Cham. & av & out & 25637 \\
\hline & Jacaranda micrantha Cham. & av & - & $\mathrm{s} / \mathrm{col}$ \\
\hline & Macfadyena unguis-cati (L.) A. H. Gentry & $\operatorname{tr}$ & out & 28387 \\
\hline & Pyrostegia venusta (Ker-Gawl.) Miers & $\operatorname{tr}$ & - & s/col. \\
\hline & Pithecoctenium crucigerum (L.) A. H. Gentry & $\operatorname{tr}$ & nov & 28314 \\
\hline Boraginaceae & Cordia axillaris I. M. Johnst. & $a b$ & $\operatorname{dez}$ & 29310 \\
\hline Brassicaceae & Cardamine chenopodifolia Pers. & he & set & 28390 \\
\hline Bromeliaceae & Aechmea distichanta Lem. & he & out & 25709 \\
\hline Burmanniaceae & Apteria aphylla (Nutt.) Barnhart ex Small & he & $\operatorname{dez}$ & 29313 \\
\hline \multirow[t]{2}{*}{ Caesalpiniaceae } & Bauhinia microstachya (Raddi) J. F. Macbr. & $\operatorname{tr}$ & $\operatorname{dez}$ & 29447 \\
\hline & Senna multijuga (Rich.) Irwin e Barneby & av & - & $\mathrm{s} / \mathrm{col}$ \\
\hline \multirow[t]{2}{*}{ Cannaceae } & Canna indica $\mathrm{L}$. & he & ago & 28391 \\
\hline & Canna confusa Richardson e L. B. Sm. & he & jul & 28109 \\
\hline Canellaceae & Capsicodendron dinisii (Schwacke) Occhioni & av & - & $\mathrm{s} / \mathrm{col}$ \\
\hline Caprifoliaceae & Lonicera japonica Thunb. & $\mathrm{ab}$ & out & 25638 \\
\hline \multirow[t]{4}{*}{ Celastraceae } & Maytenus alaternoides Reissek & av & - & $\mathrm{s} / \mathrm{col}$ \\
\hline & Maytenus aquifolium Mart. & av & - & s/col. \\
\hline & Maytenus evonymoides Reissek & $\mathrm{ab}$ & jul & 26041 \\
\hline & Maytenus ilicifolia (Schrad.) Planch. & av & - & s/col. \\
\hline Clethraceae & Clethra scabra Pers. & av & - & $\mathrm{s} / \mathrm{col}$. \\
\hline \multirow[t]{4}{*}{ Commelinaceae } & Commelina sp. & he & jan & 39659 \\
\hline & Dichorisandra hexandra (Aubl.) Standl. & $\operatorname{tr}$ & jan & 29665 \\
\hline & Tradescantia fluminensis Vell. & he & nov & 29664 \\
\hline & Tradescantia sellowiana Kunth & he & fev & 29817 \\
\hline \multirow[t]{2}{*}{ Convolvulaceae } & Dichondra macrocalyx Meisn. & he & out & 29318 \\
\hline & Dichondra repens Forster & he & out & 28393 \\
\hline \multirow[t]{5}{*}{ Cucurbitaceae } & Cayaponia cabocla (Vell.) Mart. & $\operatorname{tr}$ & jan & 40797 \\
\hline & Cucurbitaceae 1 & he & fev & 40801 \\
\hline & Cucurbitaceae 2 & $\operatorname{tr}$ & $\operatorname{dez}$ & 40798 \\
\hline & Cucurbitaceae 3 & $\operatorname{tr}$ & $\operatorname{dez}$ & 40800 \\
\hline & Cucurbitaceae 4 & $\operatorname{tr}$ & fev & 40799 \\
\hline Cunoniaceae & Lamanonia ternata Vell. & av & dez & 29815 \\
\hline Cyperaceae & Carex pseudo-cyperus L. & he & out & 29464 \\
\hline
\end{tabular}




\begin{tabular}{|c|c|c|c|c|}
\hline Famílias & Espécies & $\begin{array}{l}\text { Forma } \\
\text { de vida }\end{array}$ & Mês & UPCB \\
\hline & Carex purpureovaginata Boeck. & he & nov & 29321 \\
\hline & Carex sellowiana Schltdl. & he & set & 29322 \\
\hline & Carex sororia Kunth & he & out & 29323 \\
\hline & Carex brasiliensis A. St. Hil. & he & out & 37907 \\
\hline & Carex sp. & he & nov & 37924 \\
\hline & Rhynchospora floribunda Boeck. & he & mai & 27451 \\
\hline & Scleria hirtella Sw. & he & dez & 29325 \\
\hline \multirow[t]{3}{*}{ Dioscoreaceae } & Dioscorea sp.1 & $\operatorname{tr}$ & nov & 39658 \\
\hline & Dioscorea $\mathrm{sp} .2$ & $\operatorname{tr}$ & out & 39631 \\
\hline & Dioscorea $\mathrm{sp} .3$ & $\operatorname{tr}$ & $\operatorname{dez}$ & 39657 \\
\hline Elaeocarpaceae & Sloanea monosperma Vell. & av & - & s/col. \\
\hline Erythroxylaceae & Erythroxylum deciduum A. St.-Hil. & av & set & 26042 \\
\hline \multirow[t]{10}{*}{ Euphorbiaceae } & Acalypha communis Müll. Arg. & he & out & 30111 \\
\hline & Acalypha gracilis Spreng. & he & jan & 30112 \\
\hline & Alchornea triplinervia (Spreng.) Müll. Arg. & av & - & $\mathrm{s} / \mathrm{col}$ \\
\hline & Bernardia pulchella (Baill.) Müll. Arg. & $\mathrm{ab}$ & $\operatorname{mar}$ & 30113 \\
\hline & Chiropetalum tricoccum (Vell.) Chodat e Hassl. & he & out & 28395 \\
\hline & Dalechampia micromeria Baill. & $\operatorname{tr}$ & nov & 28546 \\
\hline & Sapium glandulatum (Vell.) Pax & av & - & s/col. \\
\hline & Sebastiania brasiliensis Spreng. & av & ago & 26077 \\
\hline & Sebastiania commersoniana (Baill.) L. B. Sm. e Downs & av & jul & 25718 \\
\hline & Tragia sellowiana (Klotzsch) Müll.Arg. & $\operatorname{tr}$ & dez & 29453 \\
\hline \multirow[t]{9}{*}{ Fabaceae } & Desmodium incanum DC. & he & nov & 29330 \\
\hline & Desmodium uncinatum (Jacq.) DC. & he & mar & 30581 \\
\hline & Erythrina falcata Benth. & av & nov & 29331 \\
\hline & $\begin{array}{l}\text { Lonchocarpus cultratus (Vell.) A. M. G. Azevedo e H. C. } \\
\text { Lima }\end{array}$ & av & - & s/col. \\
\hline & Lonchocarpus subglaucescens Mart. ex Benth. & av & jan & 27128 \\
\hline & Machaerium brasiliense Vogel & av & - & s/col. \\
\hline & Machaerium paraguariense Hassl. & av & - & $\mathrm{s} / \mathrm{col}$ \\
\hline & Machaerium stipitatum (DC.) Vogel & av & - & $\mathrm{s} / \mathrm{col}$ \\
\hline & Phaseolus caracalla L. & $\operatorname{tr}$ & dez & 27129 \\
\hline \multirow[t]{7}{*}{ Flacourtiaceae } & Banara parviflora (A. Gray) Benth. & av & - & s/col. \\
\hline & Banara tomentosa Clos & av & - & s/col. \\
\hline & Casearia decandra Jacq. & av & out & 28316 \\
\hline & Casearia obliqua Spreng. & av & jan & 25997 \\
\hline & Casearia sylvestris $\mathrm{Sw}$. & av & jul & 25644 \\
\hline & Xylosma ciliatifolium Eichler & av & out & 25719 \\
\hline & Xylosma prockia (Turcz.) Turcz. & av & - & s/col. \\
\hline Iridaceae & Crocosmia crocosmiflora (Nicholson) N. E. Br. & he & jan & 26082 \\
\hline \multirow{4}{*}{ Lamiaceae } & Hyptis heterodon Epling & he & fev & 39667 \\
\hline & Ocimum selloi Benth. & he & fev & 27126 \\
\hline & Salvia guaranitica A.St.-Hil. ex Benth. & he & nov & 28560 \\
\hline & Salvia melissiflora Benth. & he & mar & 27127 \\
\hline \multirow[t]{11}{*}{ Lauraceae } & Cinnamomum amoenum (Nees) Kosterm. & av & fev & 30580 \\
\hline & Cinnamomum sp. & av & - & $\mathrm{s} / \mathrm{col}$ \\
\hline & Cryptocarya aschersoniana $\mathrm{Mez}$ & av & nov & 28558 \\
\hline & Nectandra lanceolata Nees e Mart. ex Nees & av & - & $\mathrm{s} / \mathrm{col}$ \\
\hline & Nectandra megapotamica (Spreng.) Mez & av & ago & 28323 \\
\hline & Ocotea corymbosa (Meisn.) Mez & av & - & s/col. \\
\hline & Ocotea nutans (Nees) Mez & av & $\operatorname{mar}$ & 27456 \\
\hline & Ocotea odorifera (Vell.) Rohwer & av & - & $\mathrm{s} / \mathrm{col}$ \\
\hline & Ocotea puberula (Rich.) Nees & av & - & $\mathrm{s} / \mathrm{col}$ \\
\hline & Ocotea pulchella (Nees) Mez & av & out & 27455 \\
\hline & Persea major (Nees) L. E. Kopp & av & nov & 29343 \\
\hline Liliaceae & Cordyline spectabilis Kunth et Bouché & $\mathrm{ab}$ & nov & 29344 \\
\hline Loganiaceae & Strychnos brasiliensis (Spreng.) Mart. & av & nov & 29345 \\
\hline
\end{tabular}




\begin{tabular}{|c|c|c|c|c|}
\hline Famílias & Espécies & $\begin{array}{l}\text { Forma } \\
\text { de vida }\end{array}$ & Mês & UPCB \\
\hline \multirow{3}{*}{ Loranthaceae } & Phrygilanthus acutifolius (Ruiz e Pav.) Eichler & hp & mai & 27458 \\
\hline & Struthanthus polyrhizus Mart. & hp & ago & 28402 \\
\hline & Struthanthus vulgaris Mart. & hp & out & 28542 \\
\hline \multirow[t]{2}{*}{ Lythraceae } & Heimia myrtifolia Cham. e Schltdl. & he & jan & 25999 \\
\hline & Lafoensia pacari A. St.-Hil. & av & dez & 29347 \\
\hline \multirow{2}{*}{ Malpighiaceae } & Heteropterys intermedia (A. Juss.) Griseb. & $\operatorname{tr}$ & jan & 27473 \\
\hline & Heteropterys martiana A. Juss. & $\operatorname{tr}$ & jan & 30301 \\
\hline \multirow[t]{4}{*}{ Malvaceae } & Pavonia schrankii Spreng. & $a b$ & out & 27132 \\
\hline & Pavonia sepium A. St.-Hil. & $a b$ & jan & 29457 \\
\hline & Sida carpinifolia L. f. & $\mathrm{ab}$ & mar & 30120 \\
\hline & Wissadula parviflora (A. St.-Hil.) R. E. Fr. & he & mar & 27134 \\
\hline \multirow{11}{*}{ Melastomatacea } & Leandra australis (Cham.) Cogn. & he & jun & 27459 \\
\hline & Leandra refracta Cogn. & $\mathrm{ab}$ & out & 28406 \\
\hline & Leandra regnellii (Triana) Cogn. & $\mathrm{ab}$ & out & 28404 \\
\hline & Leandra sublanata Cogn. & $\mathrm{ab}$ & mai & 25273 \\
\hline & Leandra xanthocoma (Naudin) Cogn. & he & dez & 29350 \\
\hline & Miconia cinerascens Miq. & av & nov & 30299 \\
\hline & Miconia hyemalis A. St.-Hil. e Naudin ex Naudin & av & ago & 28409 \\
\hline & Miconia petropolitana Cogn. & av & set & 28411 \\
\hline & Miconia sellowiana Naudin & av & set & 28413 \\
\hline & Tibouchina clinopodifolia (DC.) Cogn. & he & $\mathrm{abr}$ & 27135 \\
\hline & Tibouchina sellowiana (Cham.) Cogn. & av & fev & 26085 \\
\hline \multirow[t]{3}{*}{ Meliaceae } & Cabralea canjerana (Vell.) Mart. & av & out & 27136 \\
\hline & Cedrela fissilis Vell. & av & - & $\mathrm{s} / \mathrm{col}$ \\
\hline & Cedrela cf. odorata L. & av & - & $\mathrm{s} / \mathrm{col}$. \\
\hline Menispermaceae & Cissampelos pareira $\mathrm{L}$. & $\operatorname{tr}$ & out & 29803 \\
\hline \multirow[t]{3}{*}{ Mimosaceae } & Acacia nitidifolia Speg. & $\operatorname{tr}$ & nov & 30121 \\
\hline & Inga virescens Benth. & av & jan & 29805 \\
\hline & Mimosa scabrella Benth. & av & jun & 27402 \\
\hline \multirow[t]{2}{*}{ Monimiaceae } & Mollinedia clavigera Tul. & $a b$ & jul & 26086 \\
\hline & Mollinedia schottiana (Spreng.) Perk. & $\mathrm{ab}$ & nov & 29352 \\
\hline \multirow[t]{3}{*}{ Moraceae } & Ficus enormis (Mart. ex Miq.) Miq. & av & mai & 29354 \\
\hline & Ficus luschnathiana (Miq.) Miq. & av & jan & 27137 \\
\hline & Sorocea bonplandii (Baill.) W. C. Burger, Lanj. e Wess. Boer & av & - & $\mathrm{s} / \mathrm{col}$ \\
\hline \multirow[t]{5}{*}{ Myrsinaceae } & Rapanea ferruginea (Ruiz e Pav.) Mez & av & mar & 30297 \\
\hline & Rapanea lancifolia $\mathrm{Mez}$ & av & - & $\mathrm{s} / \mathrm{col}$ \\
\hline & Rapanea umbellata (Mart.) Mez & av & ago & 25747 \\
\hline & Rapanea sp.1 & av & out & 39654 \\
\hline & Rapanea sp.2 & av & set & 39655 \\
\hline \multirow[t]{17}{*}{ Myrtaceae } & Blepharocalyx salicifolius (Kunth) O. Berg & av & - & $\mathrm{s} / \mathrm{col}$ \\
\hline & Calycorectes australis D. Legrand & av & - & $\mathrm{s} / \mathrm{col}$ \\
\hline & Calyptranthes concinna DC. & av & set & 28327 \\
\hline & Campomanesia xanthocarpa O. Berg & av & jul & 25748 \\
\hline & Eugenia obovata Poir. & av & - & s/col. \\
\hline & Eugenia platysema $\mathrm{O}$. Berg & av & $\mathrm{abr}$ & 30655 \\
\hline & Eugenia uniflora $\mathrm{L}$. & av & $\mathrm{abr}$ & 27139 \\
\hline & Mosiera prismatica (D. Legrand) Landrum & av & jan & 27138 \\
\hline & Myrceugenia euosma (O. Berg) D. Legrand & av & jan & 26088 \\
\hline & Myrceugenia miersiana (Gardner) D. Legrand e Kausel & av & abr & 27141 \\
\hline & Myrceugenia myrcioides (Cambess.) Berg & av & ago & 39627 \\
\hline & $\begin{array}{l}\text { Myrceugenia ovata (Hook. e Arn.) O. Berg var. gracilis } \\
\text { (Burret) Landrum }\end{array}$ & $a b$ & abr & 27144 \\
\hline & Myrcia hatschbachii D. Legrand & av & nov & 29357 \\
\hline & Myrcia laruotteana Cambess. & av & out & 27140 \\
\hline & Myrcia multiflora (Lam.) DC. & av & nov & 29358 \\
\hline & Myrcia obtecta (Berg) Kiaersk. & av & nov & 28559 \\
\hline & Myrcia rostrata DC. & av & - & $\mathrm{s} / \mathrm{col}$ \\
\hline
\end{tabular}




\begin{tabular}{|c|c|c|c|c|}
\hline Famílias & Espécies & $\begin{array}{l}\text { Forma } \\
\text { de vida }\end{array}$ & Mês & UPCB \\
\hline & Myrcia venulosa DC. & av & $\operatorname{dez}$ & 39646 \\
\hline & Myrcia sp. & av & - & s/col. \\
\hline & Myrcianthes gigantea (D. Legrand) D. Legrand & av & jan & 27145 \\
\hline & Myrciaria tenella (DC.) O. Berg & $\mathrm{ab}$ & mar & 30122 \\
\hline & Myrrhinium atropurpureum Schott var. octandrum Benth. & av & ago & 28331 \\
\hline & Pimenta pseudocaryophyllus (Gomes) Landrum & av & - & s/col. \\
\hline & Psidium cattleyanum Sabine & av & $\operatorname{mar}$ & 30294 \\
\hline & Psidium longipetiolatum D. Legrand & av & mar & 30293 \\
\hline Nyctaginaceae & Guapira opposita (Vell.) Reitz & av & - & $\mathrm{s} / \mathrm{col}$ \\
\hline \multirow[t]{4}{*}{ Orchidaceae } & Cyclopogon elegans Hoehne & he & ago & 29459 \\
\hline & Erythrodes austrobrasiliensis (Porsch) Pabst & he & mar & 26046 \\
\hline & Galeandra beyrichii Rchb.f. & he & fev & 28504 \\
\hline & Mesadenella cuspidata (Lindl. e Rchb.f.) Garay & he & mai & 27436 \\
\hline \multirow{2}{*}{ Oxalidaceae } & Oxalis debilis Humb., Bonpl. e Kunth & he & out & 28419 \\
\hline & Oxalis linarantha Lourteig & he & set & 28332 \\
\hline \multirow[t]{6}{*}{ Passifloraceae } & Passiflora actinia Hook. & $\operatorname{tr}$ & out & 25655 \\
\hline & Passiflora alata Dryand. & $\operatorname{tr}$ & nov & 28538 \\
\hline & Passiflora amethystina J. C. Mikan & $\operatorname{tr}$ & dez & 29673 \\
\hline & Passiflora caerulea L. & $\operatorname{tr}$ & - & s/col. \\
\hline & Passiflora edulis Sims & $\operatorname{tr}$ & nov & 28556 \\
\hline & Passiflora organensis Gardner & $\operatorname{tr}$ & mar & 30124 \\
\hline Phytolaccaceae & Petiveria alliacea $\mathrm{L}$. & he & fev & 26054 \\
\hline \multirow[t]{5}{*}{ Piperaceae } & Peperomia caulibarbis Miq. & he & $\operatorname{mar}$ & 28042 \\
\hline & Peperomia hilariana Miq. & he & $\operatorname{mar}$ & 28123 \\
\hline & Piper gaudichaudianum Kunth & $\mathrm{ab}$ & ago & 28043 \\
\hline & Piper xylosteoides (Kunth) Steud. & $\mathrm{ab}$ & jul & 27121 \\
\hline & Piper mikanianum (Kunth) Steud. & he & out & 29801 \\
\hline \multirow[t]{17}{*}{ Poaceae } & Festuca ulochaeta Nees ex Steud. & he & fev & 29796 \\
\hline & Ichnanthus cf. ruprechtii Döll & he & $\operatorname{mar}$ & 31858 \\
\hline & Ichnanthus pallens (Sw.) Munro ex Benth. & he & abr & 37913 \\
\hline & Ichnanthus tenuis (Presl) Hitch. e Chase & he & jan & 37914 \\
\hline & Oplismenus setarius (Lam.) Roem. e Schult. & he & jan & 27403 \\
\hline & Panicum glutinosum Sw. & he & $\operatorname{mar}$ & 29800 \\
\hline & Panicum pantrichum Hack. & he & mai & 30127 \\
\hline & Panicum pilosum $\mathrm{Sw}$. & he & fev & 29799 \\
\hline & Panicum rude Nees & he & nov & 29468 \\
\hline & Panicum schwackeanum Mez & he & nov & 37908 \\
\hline & Pennisetum latifolium Spreng. & he & $\operatorname{mar}$ & 30129 \\
\hline & Pharus glaber Humb., Bonpl. e Kunth & he & jan & 29367 \\
\hline & $\begin{array}{l}\text { Pseudechinolaena polystachya (Humb., Bonpl. e Kunth) } \\
\text { Stapf }\end{array}$ & he & jan & 27404 \\
\hline & Setaria poiretiana (Schult.) Kunth & he & $\operatorname{mar}$ & 29669 \\
\hline & Sorghastrum scaberrimum (Nees) Herter & he & mar & 37910 \\
\hline & Sporobolus indicus (L.) R. Br. & he & jan & 29471 \\
\hline & Sporobolus pseudairoides Parodi & he & jan & 29472 \\
\hline Polygalaceae & Polygala lancifolia A. St.-Hil. e Moq. & he & jun & 27405 \\
\hline Proteaceae & Roupala brasiliensis Klotzsch & av & - & s/col. \\
\hline \multirow[t]{3}{*}{ Rhamnaceae } & Hovenia dulcis Thunb. & av & nov & 29374 \\
\hline & Rhamnus sphaerosperma Sw. & av & $\operatorname{dez}$ & 29375 \\
\hline & Scutia buxifolia Reissek & av & out & 28537 \\
\hline \multirow[t]{7}{*}{ Rosaceae } & Eriobotrya japonica Lindl. & av & $\operatorname{mar}$ & 27146 \\
\hline & Prunus brasiliensis (Cham. e Schltd1.) D. Dietr. & av & jul & 25307 \\
\hline & Prunus myrtifolia (L.) Urb. & av & set & 26058 \\
\hline & Prunus sellowii Koehne & av & jan & 26059 \\
\hline & Rubus brasiliensis Mart. & $\mathrm{ab}$ & mai & 27410 \\
\hline & Rubus erythroclados Mart. ex Hook.f. & $\mathrm{ab}$ & $\mathrm{abr}$ & 27411 \\
\hline & Rubus rosaefolius J. Sm. & $\mathrm{ab}$ & jul & 34085 \\
\hline Rubiaceae & Cordiera concolor (Cham.) O. Kuntze & av & jan & 27147 \\
\hline
\end{tabular}




\begin{tabular}{|c|c|c|c|c|}
\hline Famílias & Espécies & $\begin{array}{l}\text { Forma } \\
\text { de vida }\end{array}$ & Mês & UPCB \\
\hline & Coccocypselum guianense (Aubl.) K. Schum & he & mai & 27415 \\
\hline & Coccocypselum lanceolatum (Ruiz e Pav.) Pers. & he & mai & 27148 \\
\hline & Coccocypselum cordifolium Nees e Mart. & he & nov & 28554 \\
\hline & Guettarda uruguensis Cham. e Schltdl. & av & nov & 29376 \\
\hline & Manettia luteo-rubra (Vell.) Benth. & $\operatorname{tr}$ & out & 25752 \\
\hline & Psychotria longipes Müll. Arg. & av & - & s/col. \\
\hline & Psychotria vellosiana Benth. & av & jun & 28338 \\
\hline & Psychotria suterella Müll. Arg. & $a b$ & mar & 27416 \\
\hline & Randia armata (Sw.) DC. & av & nov & 28533 \\
\hline & Relbunium hypocarpium (L.) Hemsl. & he & ago & 28339 \\
\hline & Rudgea jasminioides (Cham.) Müll. Arg. & $\mathrm{ab}$ & out & 26093 \\
\hline & Rudgea parquioides (Cham.) Müll. Arg. & $\mathrm{ab}$ & fev & 27149 \\
\hline \multirow[t]{3}{*}{ Rutaceae } & Pilocarpus pennatifolius Lem. & av & jan & 26097 \\
\hline & Zanthoxylum kleinii (R. S. Cowan) P. G. Waterman & av & - & $\mathrm{s} / \mathrm{col}$ \\
\hline & Zanthoxylum rhoifolium Lam. & av & out & 25754 \\
\hline \multirow[t]{9}{*}{ Sapindaceae } & Allophylus edulis (A. St.-Hil., Cambess. e A. Juss.) Radlk. & av & set & 25658 \\
\hline & $\begin{array}{l}\text { Allophylus guaraniticus (A. St.-Hil.) Radlk. var. pilosus } \\
\text { Barkley e Villa }\end{array}$ & av & jul & 25660 \\
\hline & Cupania vernalis Cambess. & av & - & $\mathrm{s} / \mathrm{col}$ \\
\hline & Matayba elaeagnoides Radlk. & av & out & 28563 \\
\hline & Paullinia carpopodea Cambess. & $\operatorname{tr}$ & mai & 27429 \\
\hline & Serjania caracasana (Jacq.) Willd. & $\operatorname{tr}$ & jun & 25756 \\
\hline & Serjania gracilis Radlk. & $\operatorname{tr}$ & abr & 27430 \\
\hline & Serjania laruotteana Cambess. & $\operatorname{tr}$ & jan & 27152 \\
\hline & Serjania multiflora Cambess. & $\operatorname{tr}$ & jun & 25663 \\
\hline Simaroubaceae & Picramnia parvifolia Engl. & av & out & 28584 \\
\hline \multirow{2}{*}{ Smilacaceae } & Smilax cognata Kunth & $\operatorname{tr}$ & out & 30291 \\
\hline & Smilax cf. spinosa Mill. & $\operatorname{tr}$ & nov & 39373 \\
\hline \multirow{25}{*}{ Solanaceae } & Vassobia breviflora (Sendtn.) Hunz. & $\mathrm{ab}$ & out & 26098 \\
\hline & Brunfelsia pauciflora (Cham. e Schltdl.) Benth. & $\mathrm{ab}$ & out & 25308 \\
\hline & Brunfelsia uniflora (Pohl) D. Don & $a b$ & out & 25664 \\
\hline & Capsicum praetermissum Heiser e P. G. Sm. & $a b$ & mai & 27426 \\
\hline & Capsicum cf. villosum Sendtn. & $a b$ & out & 39375 \\
\hline & Cestrum amictum Schltdl. & $a b$ & jul & 25309 \\
\hline & Cestrum intermedium Sendtn. & $a b$ & mar & 28048 \\
\hline & Cyphomandra corymbiflora Sendtn. & he & set & 28344 \\
\hline & Cyphomandra diploconos (Mart.) Sendtn. & $\mathrm{ab}$ & out & 25757 \\
\hline & Cyphomandra divaricata (Mart.) Sendtn. & $\mathrm{ab}$ & nov & 29385 \\
\hline & Cyphomandra cf . premnifolia (Miers) Dunal & $\mathrm{ab}$ & dez & 39643 \\
\hline & Physalis pubescens L. & he & mar & 27158 \\
\hline & Solanum acerosum Sendtn. & $\mathrm{ab}$ & jan & 26103 \\
\hline & Solanum affine Sendtn. & he & jan & 28049 \\
\hline & Solanum americanum Mill. & he & jan & 26105 \\
\hline & Solanum bullatum Vell. & av & - & s/col. \\
\hline & Solanum caeruleum Vell. & $a b$ & jan & 28051 \\
\hline & Solanum gemellum Mart. ex Sendtn. & $a b$ & mai & 25310 \\
\hline & Solanum granuloso-leprosum Dunal & $a b$ & $\mathrm{abr}$ & 28046 \\
\hline & Solanum inodorum Vell. & $\operatorname{tr}$ & set & 25311 \\
\hline & Solanum megalochiton Mart. & $a b$ & ago & 28587 \\
\hline & Solanum microrbitum L. B. Sm. e Downs & $a b$ & mar & 28052 \\
\hline & Solanum pseudoquina A. St.-Hil. & av & jan & 26114 \\
\hline & Solanum sanctae-catharinae Dunal & av & nov & 28586 \\
\hline & Solanum swartzianum Roem. e Schult. & av & mai & 25312 \\
\hline \multirow{2}{*}{ Styracaceae } & Styrax leprosus Hook. e Arn. & av & nov & 28552 \\
\hline & Styrax martii Seub. & av & - & s/col. \\
\hline \multirow[t]{3}{*}{ Symplocaceae } & Symplocos celastrinea Mart. ex Miq. & av & - & $\mathrm{s} / \mathrm{col}$ \\
\hline & Symplocos tetrandra Mart. ex Miq. & av & jun & 27425 \\
\hline & Symplocos uniflora (Pohl) Benth. & av & out & 25759 \\
\hline
\end{tabular}




\begin{tabular}{|c|c|c|c|c|}
\hline Famílias & Espécies & $\begin{array}{l}\text { Forma } \\
\text { de vida }\end{array}$ & Mês & UPCB \\
\hline & Symplocos sp. & av & - & s/col. \\
\hline Thymelaeaceae & Daphnopsis racemosa Griseb. & $\mathrm{ab}$ & ago & 28347 \\
\hline \multirow[t]{2}{*}{ Tiliaceae } & Luehea divaricata Mart. e Zucc. & av & jan & 26006 \\
\hline & Triumfetta semitriloba Jacq. & he & mar & 26123 \\
\hline Tropaeolaceae & Tropaeolum pentaphyllum Lam. & $\operatorname{tr}$ & set & 28348 \\
\hline Ulmaceae & Celtis tala Gillies ex Planch. & av & dez & 29387 \\
\hline \multirow[t]{2}{*}{ Urticaceae } & Pilea pubescens Liebm. & he & mar & 27431 \\
\hline & Urera baccifera (L.) Gaudich. & $\mathrm{ab}$ & $\operatorname{dez}$ & 26124 \\
\hline \multirow[t]{2}{*}{ Valerianaceae } & Valeriana salicariifolia Vahl & he & out & 29481 \\
\hline & Valeriana scandens Loefl. et L. & $\operatorname{tr}$ & jun & 27432 \\
\hline \multirow[t]{6}{*}{ Verbenaceae } & Aegiphila sellowiana Cham. & av & mar & 27160 \\
\hline & Citharexylum solanaceum Cham. & av & nov & 29388 \\
\hline & Duranta vestita Cham. & av & out & 25766 \\
\hline & Lantana brasiliensis Link & $\mathrm{ab}$ & dez & 26125 \\
\hline & Lantana camara $\mathrm{L}$. & $\mathrm{ab}$ & jan & 26127 \\
\hline & Lantana cf. fucata Lindl. & $\mathrm{ab}$ & nov & 39664 \\
\hline \multirow[t]{2}{*}{ Violaceae } & Anchietea pyrifolia (Mart.) G. Don & $\operatorname{tr}$ & out & 27163 \\
\hline & Viola cerasifolia A.St.-Hil. & he & nov & 29392 \\
\hline Vitaceae & Cissus verticillata (L.) Nicolson e C. E. Jarvis & $\operatorname{tr}$ & jan & 29794 \\
\hline Winteraceae & Drimys brasiliensis Miers & av & $\operatorname{dez}$ & 26007 \\
\hline
\end{tabular}

\section{DISCUSSÃO}

A vegetação nativa do Parque, representada pelo fragmento de FOM, encontra-se bem conservada, tanto por esforços da Secretaria de Estado do Meio Ambiente e da Polícia Florestal, que desenvolvem atividades de fiscalização, como pela própria comunidade que, gradativamente, está se conscientizando da importância da conservação do ambiente. Os resultados florísticos obtidos com a realização deste trabalho evidenciam tal fato.

A área apresentou elevada diversidade de espécies quando comparada a outros trabalhos que foram realizados em Curitiba, como os de Cervi et al. (1987a, 1987b, 1989), Roseira (1990) e Dombrowski e Kuniyoshi (1967). Em todos esses trabalhos, a diversidade foi menor do que aquela obtida para o Parque Barigüi. Em parte, isto pode ser justificado pelo fato de terem sido realizados em áreas com extensões menores ( 1 a 7 ha, aproximadamente) ou por terem limitado a amostragem, considerando espécies com apenas uma forma de vida ou pertencentes a um único grupo de plantas, por exemplo, pteridófita.

Para alguns autores, como Cervi et al. (1987b), a interferência antrópica no ambiente, sob diferentes formas, como o depósito de detritos e a presença de espécies exóticas e/ou ruderais, também representa um fator que pode estar relacionado à baixa diversidade de espécies em levantamentos florísticos. Quanto a esse aspecto, no Parque Barigüi também foram identificadas algumas espécies exóticas, porém de forma pouco representativa e sem interferência na estrutura da vegetação, conforme observado em trabalho fitossociológico realizado na mesma área (KOZERA et al., inédito). A introdução dessas espécies na área provavelmente ocorreu em função da intensa circulação de pessoas e de aves com hábitos urbanos, que podem, com isso, ter contribuído para a dispersão das espécies citadas.

Com relação à interferência das espécies exóticas na estrutura da vegetação, situação contrária à do Parque Barigüi foi registrada por Roseira (1990) em estudo fitossociológico realizado em um fragmento de FOM localizado em um bosque de Curitiba. Dentre os resultados obtidos, o alfeneiro (Ligustrum lucidum W. T. Aiton, Oleaceae), espécie arbórea exótica, destacou-se como a mais importante na amostragem, evidenciando forte interferência no ambiente através da descaracterização da estrutura da vegetação original.

Importante mencionar que as comparações florísticas entre o Parque Barigüi e outras áreas florestais foram limitadas em função da inexistência de levantamentos realizados de forma extensiva e que tivessem incluído espécies da floresta com todas as formas de vida. Como exceção, cita-se o trabalho de Britez et al. (1995), muito semelhante em termos metodológicos ao presente estudo realizado no Parque Barigüi. 
Britez et al. (1995) realizaram levantamento em área localizada em São Mateus do Sul, município próximo a Curitiba. Observaram FOM conservada e vegetação arbórea secundária, e incluíram todas as formas de vida. Dentre os resultados apresentados, listaram 307 espécies pertencentes a 76 famílias. Comparando-se esses resultados com os do Parque Barigüi, apresentados neste trabalho e que somados àqueles apresentados por Dittrich et al. (1999), referentes às epífitas da mesma área, passam a totalizar 464 espécies e 117 famílias, foram significativos porém de menor riqueza. Na floresta em São Mateus do Sul, o corte seletivo do sub-bosque, realizado em grande parte da área para beneficiar a exploração da erva-mate (Ilex paraguariensis A. St.-Hil.), comercializada na região, interfere, entre outros aspectos, na regeneração e na cobertura herbácea do sub-bosque, com conseqüente diminuição da diversidade florística. As herbáceas, eliminadas no processo de limpeza do sub-bosque, são elementos que contribuem para o incremento florístico de áreas florestais. Constituem um dos componentes mais importantes da floresta, porém são relegadas a segundo plano ou até mesmo ignoradas na maioria dos trabalhos realizados. A fim de exemplificar, no Parque Barigüi, as herbáceas corresponderam a cerca de $33 \%$ do total de espécies registradas, valor próximo daquele obtido para as arbóreas, forma de vida representada no levantamento com o maior número de espécies $(36 \%)$.

Em síntese, os resultados apresentados neste trabalho evidenciam que a vegetação nativa do Parque Barigüi tem grande riqueza de espécies vegetais vasculares, mesmo encontrando-se situada em área urbana e sob interferência antrópica. A elevada diversidade de espécies, associada à necessidade de preservação das formações vegetais do Brasil, sob acelerado processo de degradação, justificam a importância da contínua conservação dessa área, fragmento remanescente das Florestas Ombrófilas Mistas que ocorreram no passado em grande parte do município de Curitiba.

\section{AGRADECIMENTOS}

Ao CNPq (bolsas de iniciação científica), à Secretaria de Estado do Meio Ambiente de Curitiba (autorização para a realização deste trabalho), ao Departamento de Botânica/UFPR (laboratórios), a Rodrigo de Andrade Kersten (abstract) e aos amigos e familiares que colaboraram durante as atividades de campo.

\section{REFERÊNCIAS}

ABE, L. A.; BARDDAL, M. L.; BERNARDI, D. Mapeamento e caracterização da cobertura vegetal do Parque Barigüi, Curitiba, Paraná. In: "40 ANOS DE ENGENHARIA FLORESTAL - UNIVERSIDADE FEDERAL DO PARANÁ”. Pesquisa Florestal Online, Curitiba, 2000. Anais do Evento... Curitiba : UFPR/Comite de Pesquisa do Setor de Ciencias Agrarias, 2000. p.129.

BACKES, A. Dinâmica do pinheiro-brasileiro. Iheringia, série Botânica, Porto Alegre, n.30, p.49-84, 1983.

BRITEZ, R. M.; SILVA, S. M.; SOUZA, W. S.; MOTTA, J. T. W. Levantamento florístico em Floresta Ombrófila Mista, São Mateus do Sul, Paraná, Brasil. Arquivos de Biologia e Tecnologia, Curitiba, n.38, p.1147-1161, 1995.

BRUMMITT, R. K.; POWEL, C. E. Authors of plant names. Kew: Royal Botanic Gardens, 1992.

CERVI, A. C.; ACRA, L. A.; RODRIGUES, L.; TRAIN, S.; IVANCHECHEN, S. L.; MOREIRA, A. L. O. R. Contribuição ao conhecimento das pteridófitas de uma mata de Araucária, Curitiba, Paraná, Brasil. Acta Biológica Paranaense, Curitiba, n.16, p.77-85, 1987a.

CERVI, A. C.; SCHIMMELPFENG, L. C. T.; PASSOS, M. Levantamento do estrato arbóreo do Capão da Educação Física da UFPR, Curitiba, Paraná, Brasil. Estudos de Biologia, Curitiba, n.17, p.49-61, $1987 b$.

CERVI, A. C.; ACRA, L. A.; RODRIGUES, L.; GABRIEL, M. M.; LOPES, M. Contribuição ao conhecimento das plantas herbáceas de uma floresta de Araucária do primeiro planalto paranaense. Ínsula, Florianópolis, n.18, p.83-98, 1988. 
CERVI, A. C.; PACIORNIK, E. F.; VIEIRA, R. F.; MARQUES, L. C. Espécies vegetais de um remanescente de floresta de Araucária (Curitiba, BR): Estudo preliminar I. Acta Biológica Paranaense, Curitiba, n.18, p.73-114, 1989.

CESTARO, L. A.; WAECHTER, J. L.; BAPTISTA, L. R. M. Fitossociologia do estrato herbáceo da mata de Araucária da Estação Ecológica de Aracuri, Esmeralda, RS. Hoehnea, São Paulo, n.13, p.59-72, 1986.

CRONQUIST, A. The evolution and classification of flowering plants. New York, New York Botanical Garden, 1988.

DITTRICH, V. A. O.; KOZERA, C.; MENEZES-SILVA, S. Levantamento florístico dos epífitos vasculares do Parque Barigüi, Curitiba, Paraná, Brasil. Iheringia, série Botânica, Porto Alegre, n.52, p.11-22, 1999.

DOMBROWSKI, L. T. D.; KUNIYOSHI, Y. S. A vegetação do “Capão da Imbuia”. Araucariana, Série Botânica, Curitiba, n.1, p.1-18, 1967.

FONT QUER, P. Diccionario de botánica. Barcelona: Labor, 1963.

GALVÃO, F.; KUNIYOSHI, Y. S.; RODERJAN, C. V. Levantamento fitossociológico das principais associações arbóreas da Floresta Nacional de Irati - PR. Floresta, Curitiba, n.19, p.30-49, 1989.

GRAF, A. B. Tropica color cyclopedia of exotic plants and trees. 4 ed. New Jersey: Roehrs, 1992.

HATSCHBACH, G.; MOREIRA FILHO, H. Catálogo florístico do Parque de Vila Velha (Estado do Paraná - Brasil). Boletim da Universidade Federal do Paraná, Curitiba, n.28, p.1-49, 1972.

HUECK, K. Distribuição e habitat natural do pinheiro-do-Paraná (Araucaria angustifolia). Boletim da Faculdade de Filosofia e Ciências da Universidade de São Paulo, n.10, p.1-24, 1953.

IAPAR. Cartas climáticas básicas do Estado do Paraná. Londrina, 1978.

IBGE. Manual técnico da vegetação brasileira. Rio de Janeiro, 1992.

IPNI - THE INTERNATIONAL PLANT NAMES INDEX. The international plant names index database. Disponível em: <http://www.ipni.org/index.html>. Acesso em abril 2006.

JARENKOW, J. A.; BAPTISTA, L. R. M. Composição florística e estrutura da mata com Araucaria angustifolia na Estação Ecológica de Aracuri, Esmeralda, Rio Grande do Sul. Napaea, Revista de Botânica, Porto Alegre, n.3, p.9-18, 1987.

KLEIN, R. M. Ecologia da flora e vegetação do Vale do Itajaí. Sellowia, Itajaí, n.31, p.11-164, 1979.

KLEIN, R. M. O aspecto dinâmico do pinheiro brasileiro. Sellowia, Itajaí, n.12, p.17-44, 1960.

KLEIN, R. M. Observações e considerações sobre a vegetação do planalto nordeste catarinense. Sellowia, Itajaí, n.15, p.39-57, 1963.

LONGHI, S. J. A estrutura de uma floresta natural de Araucaria angustifolia (Bertol.) O. Kuntze no sul do Brasil. 198f.. Dissertação (Mestrado em Engenharia Florestal) - Setor de Ciências Agrárias, Universidade Federal do Paraná, Curitiba, 1980.

LORENZI, H.; SOUZA, H. M. Plantas ornamentais no Brasil: arbustivas, herbáceas e trepadeiras. Nova Odessa: Plantarum, 1995.

LORENZI, H. Plantas daninhas do Brasil: terrestres, aquáticas, parasitas, tóxicas e medicinais. Nova Odessa, 1982.

MACHADO, S. A.; FIGUEIREDO, D. J.; HOSOKAWA, R. T. Composição estrutural e quantitativa de uma floresta secundária do norte catarinense. Revista do Instituto Florestal, São Paulo, n.4, p.513-518, 1992. 
MARTAU, L.; AGUiAR, L. W.; SOARES, Z. F.; BUENO, O. L. Estudo florístico do Parque dos Pinheiros e Centro de Lazer e Recreação Santa Rita, município de Farroupilha, RS, Brasil. Iheringia, série Botânica, Porto Alegre, n.28, p.17-43, 1981.

$\begin{aligned} & \text { MISSOURI BOTANICAL GARDEN. Missouri Botanical Garden's VAST } \\ & \text { nomenclatural databcular Tropicos) } \\ & \text { <http:/mobot.mobot.org/W3T/Search/vast.html }>\text {. Acesso em abril } 2006 .\end{aligned}$
files.

NEGRELE, R. A. B.; SILVA, F. C. Fitossociologia de um trecho de floresta com Araucaria angustifolia (Bertol.) O. Kuntze no município de Caçador, Santa Catarina. Boletim de Pesquisas Florestais, [S.l.], n.24/25, p.37-54, 1992.

OLIVEIRA, Y. M. M.; ROTTA, E. Levantamento da estrutura horizontal de uma mata de Araucária do primeiro planalto paranaense. Boletim de Pesquisas Florestais, [S.1.], n.4, p.1-46, 1980.

PARANÁ. Guia técnico de turismo. 4 ed. Secretaria Especial do Esporte e Turismo, Curitiba, 1994.

RIZZINI, C. T.; COIMBRA FILHO, A. F.; HOUAISS, A. Ecossistemas brasileiros. Rio de Janeiro, Editora Index, 1988.

RODERJAN, C. V.; GALVÃO, F.; KUNIYOSHI, Y. S.; HATSCHBACH, G. G. As unidades fitogeográficas do Estado do Paraná. Ciência e Ambiente, Santa Maria, n.1, p.75-92, 2002.

RODRIGUES, R. R. Métodos fitossociológicos mais usados. Casa da Agricultura, Campinas, n.1, 1988.

ROSEIRA, D. S. Composição florística e estrutura fitossociológica do Bosque com Araucaria angustifolia (Bert.) O. Ktze no Parque Estadual João Paulo II, Curitiba, Paraná. 107f. Dissertação (Mestrado em Ciências Biológicas_ - Setor de Ciências Biológicas, Universidade Federal do Paraná, Curitiba. 1990.

SENNA, R. M.; WAECHTER, J. L. Pteridófitas de uma floresta com araucária. I. Formas biológicas e padrões de distribuição geográfica. Iheringia, série Botânica, Porto Alegre, n.48, p.41-58, 1997.

SILVA, F. C.; MARCONI, L. P. Fitossociologia de uma floresta com Araucária em Colombo-PR. Boletim de Pesquisas Florestais, [S.1.], n.20, p.23-38, 1990.

SILVA, J. A.; SALOMÃO, A. N.; GRIPP, A.; LEITE, E. J. Phytososiological survey in Brazil forest genetic reserve of Caçador. Plant Ecology, Dordrecht, n.133, p.1-11, 1997.

SILVA, J. A.; SALOMÃO, A. N.; NETTO, D. A. M. Natural regeneration under Araucaria angustifolia (Bert.) O. Ktze. forest in the genetic reserve of Caçador - SC. Revista Árvore, Viçosa, n.22, p.143-153, 1998.

TRYON, R. M.; TRYON, A. F. Ferns and allied plants with special reference to Tropical America. New York : Springer-Verlag, 1982.

VELOSO, H. P.; RANGEL-FILHO, A. L. R.; LIMA, I. C. A. Classificação da vegetação brasileira, adaptada a um sistema universal. Rio de Janeiro: IBGE, 1991. 